\section{Apple 'Seohong' with High Level of Flavonoids}

\author{
Jeong Hwan Hwang, Yong Uk Shin, Daeil Kim, Seong Heo ${ }^{1}$, \\ and Seong Sig Hong
}

Fruit Research Division, National Institute of Horticultural \& Herbal Science, Rural Development Administration, 475 Imok-dong, Jangan-gu, Suwon 440706, Korea

Additional index words. cultivar description, fruit breeding, Malus $\times$ domestica

'Seohong' is an early-maturing cultivar with high levels of antioxidant phytochemicals and a much longer shelf life than 'Gala'. The fruit has a greenish yellow ground color and a medium red blush over the whole fruit. It is medium to large-sized and has an excellent appearance and outstanding flavor. The flesh is extremely juicy and sweet and retains crispness and juiciness for 2 weeks at room temperature. It ripens $\approx 1$ week later than 'Tsugaru' and is harvested in late August in Korea. The tree is moderately vigorous, spreading, semispur type with productive and regular bearing. It is not subject to alternate bearing when proper thinning is performed but has some preharvest drop. A plant patent was granted in 2007 , and the cultivar will be marketed under the trade name 'Seohong' to emphasize its early maturing and red color.

\section{Origin}

The original seedling was derived from a cross between 'Tsugaru' and 'Chukwang' made in 1992 at the National Institute of Horticultural \& Herbal Science (NIHHS) in Korea. Fruit of this seedling was first observed in late Aug. 2000 and selected on the basis of its fruit quality by Dr. J.H. Hwang. The seedling was propagated by grafting on 'M.9' rootstock for further evaluation and was assigned the breeder's number 'Wonkyo Ga 33' in our breeding records. Its regional adaptability has been evaluated at eight locations from 2003 to 2004. In 2004, this selection was designated 'Seohong' and released in 2005 (Fig. 1). The pedigree of 'Seohong' is detailed in Figure 2.

\section{Description}

This description is based on Union Internationale Pour La Protection Des Obtentions Végétales test guidelines (UPOV, 2005) for apple and includes detailed descriptions of flower, fruit, and tree morphology characteristics. Measurements are based on the aver-

Received for publication 14 Jan. 2009. Accepted for publication 11 Mar. 2009.

${ }^{1}$ To whom reprint requests should be addressed; e-mailsycarus@rda.go.kr. ages of at least 10 plant specimens collected yearly over a 3-year period.

\section{Flowers}

Flowering season. 'Seohong' flowers early, typically mid-April in Korea. It blooms almost $3 \mathrm{~d}$ later than 'Tsugaru'.

Petal color. The unopened flower color at the balloon stage is light pink. At bloom, the color turns white. The petals are overlapping.

Size. 'Seohong' is medium-sized, which is similar to or slightly smaller than 'Tsugaru'.

Pollen. The pollen has been used in several crosses to produce seedlings. The results of fruit set and number of seeds after crosspollination between 'Seohong' and several cultivars are tabled in Figure 3A. 'Seohong' is semi- or fully compatible in outcrossing
Fig. 1. Fruit of 'Seohong'apple. with other cultivars by hand pollination. To reveal S-genotype of 'Seohong', polymerase chain reaction (PCR) was performed and the primers used in this study were designed by Broothaerts (2003) according to the described methods. Consequently, the S-genotype of 'Seohong' is $\mathrm{S}_{3} \mathrm{~S}_{9}$ based on PCR (Fig. 3B).

\section{Fruit}

Shape. The fruit is globose and symmetrical (ratio height/width, 5) with weak ribbing.

Size. Fruit size is medium to large and larger than 'Tsugaru'. The average fruit weight at harvest was $257 \mathrm{~g}$ over a 3-year period in comparison with $231 \mathrm{~g}$ for 'Tsugaru'.

Color. The ground color is green-yellow to yellow with a red overcolor and almost $100 \%$ coverage at full maturity. The pattern of overcolor is solid flush with narrow stripes.

Flesh. The flesh color is cream to light yellow.

Skin. The skin is very thin, smooth, and glossy at harvest, becoming slightly waxy in long-term air storage. The russet is medium to low around the stalk cavity and absent or very low on the cheek and eye basin. The lenticels are medium in size, larger than 'Tsugaru', and medium to low in density.

Pedicel. The fruit stem is of medium length and medium thickness and deeply inserted in the stem bowl. Its average length is $26.3 \mathrm{~mm}$, and thickness is $2.7 \mathrm{~mm}$ ('Tsugaru' length $23.9 \mathrm{~mm}$, thickness $1.9 \mathrm{~mm}$ ). Pedicels are slightly apt to break at maturity.
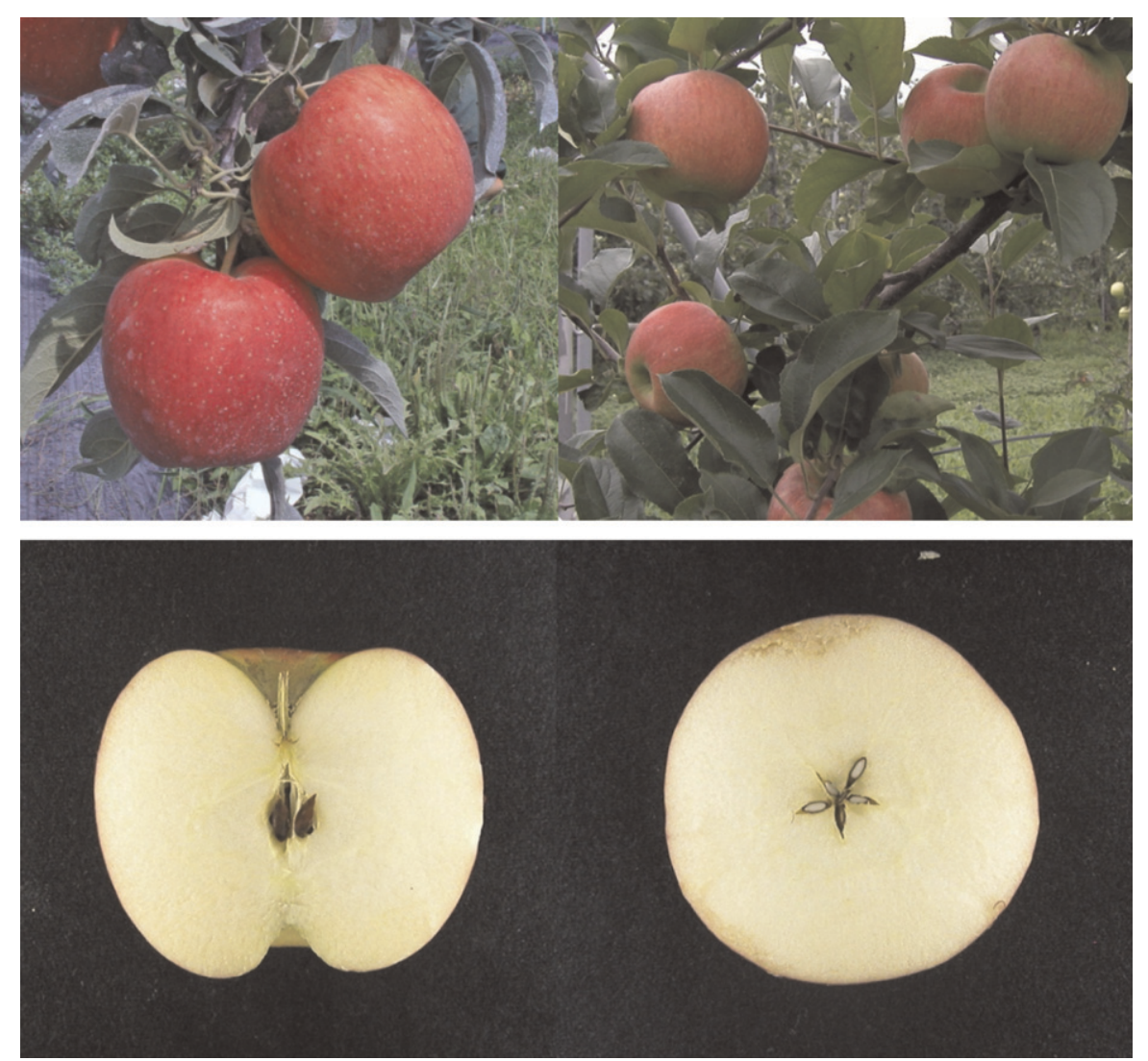


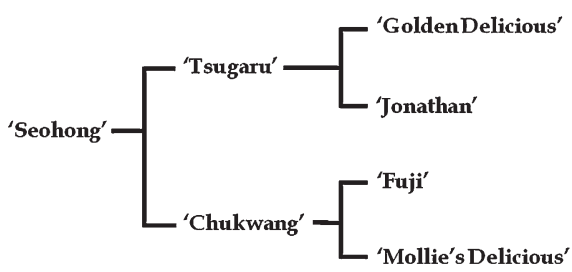

Fig. 2. Pedigree of 'Seohong'apple.

Calyx. The sepals are persistent, dry, short, and recurved. The eye is moderately opened or opened, small in size, and the basin is medium in depth. The average depth of the eye basin is $14.5 \mathrm{~mm}$ ('Tsugaru', $15.8 \mathrm{~mm}$ ) and width $37.3 \mathrm{~mm}(30.9 \mathrm{~mm})$.

Core. The core is small and median in position. Locules are partly opened, and their inner surfaces are smooth or slightly tufted. There is usually a full complement of seeds, which are acute and brown at maturity.

Texture. The fruit flesh is juicy, crisp, and moderately firm.

Firmness at harvest. Flesh firmness at harvest averaged $3.2 \mathrm{~kg} / 8 \mathrm{~mm} \varnothing$ over 3 years using a fruit pressure tester [Model FT 327 (Effegi, Alfonsine, Italy) for apple and pear].

Soluble solids and titratable acidity. At harvest, the soluble solids averaged $14.3^{\circ}$ Brix and the titratable acidity averaged $0.42 \%$ malic acid over 3 years.

Flavor. The fruit tastes sweet, delicate, and fresh.

Maturity season. In Korea, harvest season begins between the 24th and 29th of August, $\approx 1$ week later than 'Tsugaru'. Up to three harvests may be needed.

Keeping quality. 'Seohong' fruits have excellent taste, texture, and flavor. It is comparable to 'Mondial Gala' in both texture and flavor. Fruits are sweeter and more flavorful than 'Royal Gala' but are not as crisp. Its sourness is similar to 'Scarlet Gala', which is higher than 'Tsugaru'. The fruit remains fresh for 2 weeks (15 d) at room temperature, longer than 'Gala' (Fig. 4), and maintains excellent quality for $\approx 3$ to 4 months in refrigerated storage at $1{ }^{\circ} \mathrm{C}$.

Antioxidant capacity. Flavonoids are polyphenolic compounds that have antioxidant effects to protect cells against the damaging effects of reactive oxygen species such as singlet oxygen. 'Seohong' has high concentrations of flavonoids, quercetin, and rutin in fruit pulp relative to other apples (Table 1). As well, total flavonoid concentration in the flesh of 'Seohong' is higher than that of 'Jonathan', 'Pacific Gala', and 'Royal Gala' in apple flesh (data not shown; Hong, 2005).

Use. 'Seohong' is suitable to use as a dessert apple and is appropriate for mediumterm storage.

\section{Tree}

Vigor. 'Seohong' trees are moderately vigorous when grafted on 'M.9' rootstock with spreading shape. Fruits are born on spurs and shoots. Observation suggests that it is less vigorous than 'Tsugaru'. In dormant 1-year-

A

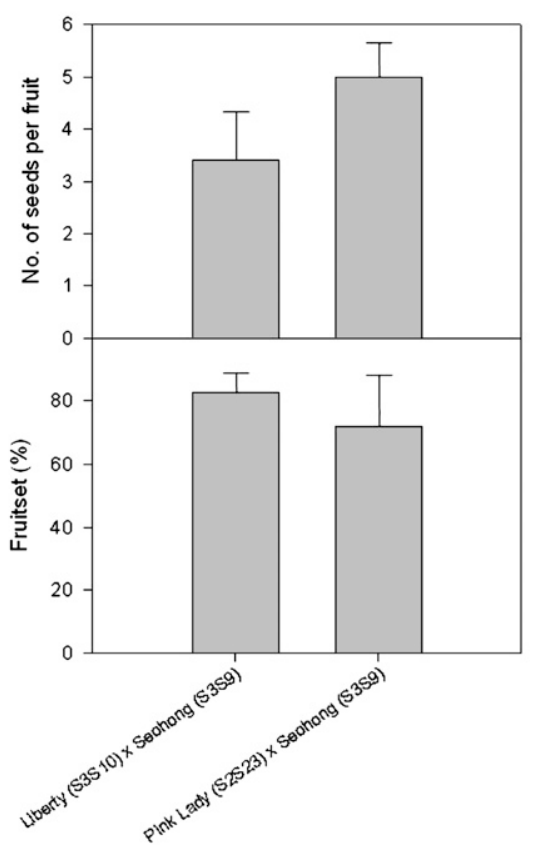

B

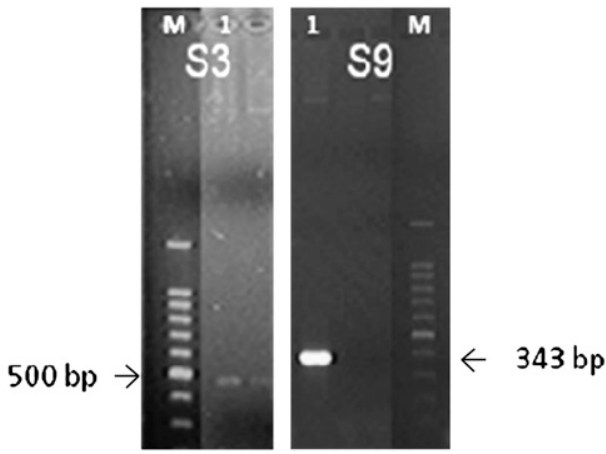

Fig. 3. (A) Fruit set and number of seeds per fruit following the crosses of 'Seohong'with other varieties. The S-genotype of cultivars is represented in parentheses. Vertical bar means SE. (B) Analysis of polymerase chain reaction fragments with S-RNase-specific primer pairs based on Broothaerts (2003). The S-genotype of 'Seohong'is $\mathrm{S}_{3} \mathrm{~S}_{9}$. M, 100 bp ladder; 1, 'Seohong'.

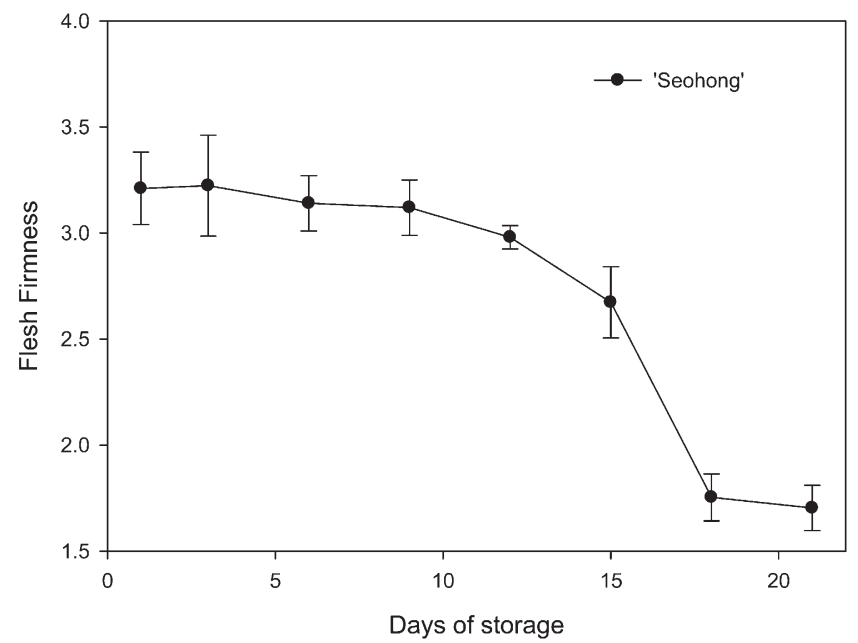

Fig. 4. Changes of flesh firmness $(\mathrm{kg} / 8 \mathrm{~mm} \varnothing)$ of 'Seohong'during storage at room temperature. The bars represent the SES of the means from three replications.

Table 1. Comparison of flavonoid contents $\left(\mu \mathrm{g} \cdot \mathrm{g}^{-1}\right.$ dry wt) of apple cultivars.

\begin{tabular}{lll}
\hline Flavonoid & \multicolumn{1}{c}{ Range } & \multicolumn{1}{c}{ Cultivar } \\
\hline Quercetin & $0-10$ & Fuji, Chohong, Himekami $(2 \mu \mathrm{g})$ \\
& $11-100$ & Chukwang, Hongro, New Jonagold, Jonathan $(85 \mu \mathrm{g})$ \\
\multirow{3}{*}{ Rutin } & Greater than 101 & Seohong, Seokwang (338 $\mu \mathrm{g})$ \\
& $0-10$ & New Jonagold, Chohong, Himekami, Jonathan $(1 \mu \mathrm{g})$ \\
& $11-100$ & Hongro, Seokwang, Fuji (15 $\mu \mathrm{g})$ \\
& Greater than 101 & Seohong, Hwahong (101 $\mu \mathrm{g})$ \\
\hline
\end{tabular}

old shoots, the thickness is $4.8 \mathrm{~mm}$ ('Tsugaru', $4.5 \mathrm{~mm}$ ), the length of the internode $3.23 \mathrm{~cm}$ ('Tsugaru', $3.08 \mathrm{~cm}$ ), and the number of lenticels per internode 55 ('Tsugaru', 48). The pubescence of 1-year-old shoot is medium to strong and is slightly less than 'Tsugaru'.

Shape. The crown is rounded and spreading.

Bearing habit. 'Seohong' bears abundant flower buds on 1-year-old branches. However, 
it does not typically flower on older branches. Heavy fruit set is typical, both number of fruits per cluster and number of clusters. It is not subject to alternate bearing if properly thinned but has some preharvest drop.

Productivity. The productivity of trees on 'M.9' is similar to that of 'Tsugaru'.

Thinning. 'Seohong' requires moderate to heavy thinning because it is prone to overset. Thinning is essential to achieve commercially acceptable fruit size in Korea. Hand thinning is common practice and accounts for $19 \%$ of labor input for apple production in Korea. Our recommendation is to thin to single king fruits $20 \mathrm{~cm}$ apart.

Disease resistance. 'Seohong' is partially resistant to white rot and bitter rot but is susceptible to Alternaria blotch (caused by Alternaria mali Roberts) according to the field tests for 3 years. However, apple scab [caused by Venturia inaequalis (Cke.) Wint.] incidence is low under conventional management in South Korea. No natural outbreaks of fire blight [caused by Erwinia amylovora (Burr.) Winslow] affected 'Seohong' during 8 years in the fields at NIHHS. Further testing is needed to better characterize the cultivar's resistance to apple scab and fire blight.

\section{Leaves}

Leaf blade. Attitude in relation to shoot of 'Seohong' is upward, the length is $8.2 \mathrm{~cm}$ ('Tsugaru', $8.9 \mathrm{~cm}$ ), and the width is $5.5 \mathrm{~cm}$ ('Tsugaru', $5.8 \mathrm{~cm}$ ). Leaf margins are serrate, and the petiole length averaged $2.7 \mathrm{~cm}$ ('Tsugaru', $2.9 \mathrm{~cm}$ ).

\section{Cultural Notes}

'Seohong' is an early-ripening cultivar similar to 'Tsugaru'. It sets heavy annual crops and must be thinned properly to attain commercial fruit size in Korea. 'Seohong' is recommended for growing in areas where average summer temperatures (below 18 to $24{ }^{\circ} \mathrm{C}$ ) are cool, because high temperatures cause discoloration of the fruit. The fruit can usually be harvested in several picks. However, 'Seohong' is somewhat susceptible to preharvest drop and should therefore be harvested at the proper season. Fruits are ready to harvest when the ground color changes from green to green-yellow and it develops a red overcolor. Lownitrogen fertilization is recommended to avoid poor coloring and to maintain proper tree vigor. 'Seohong' is expected to replace 'Tsugaru' as the main early-ripening apple cultivar in Korea owing to its better fruit quality.

\section{Availability}

'Seohong' is licensed in Korea by the Rural Development Administration and is presently available from Sunplus (Korea Fruit Agricultural Co., Federation, phone +82-02-6300-2292). 'Seohong' is protected by Korea Plant Patent No. 27.

\section{Literature Cited}

Broothaerts, W. 2003. New findings in apple Sgenotype analysis resolve previous confusion and request the re-numbering of some S-alleles. Theor. Appl. Genet. 106:703-714.

Hong, S.S. 2005. Determination of functional compounds and physiological activity in fruit of apple (Malus spp.) and pear (Pyrus spp.). Korea Univ., Seoul, Korea. PhD Diss.

The International Union for the Protection of New Varieties of Plants (UPOV). 2005. Guidelines for the conduct of tests for distinctness, uniformity, and stability. 